\title{
飞行器运动控制建模及红外辐射图像仿真
}

\author{
刘勇求 ${ }^{1}$ ，金忠庆 ${ }^{2}$ \\ (广东科技学院，东莞 523083；2. 空军航空大学，吉林 长春 130022)
}

\begin{abstract}
摘要: 实时生成飞行器在飞行过程中的红外图像对于研究飞行器的红外特性具有重要意义。飞行器在 飞行过程中, 由于其飞行速度、高度、姿态等参数变化性较大, 且机体本身容易受到环境、背景等辐 射的影响, 因此较难准确地仿真机体的温度场分布。由于飞行器机体温度场分布与飞机的运动状态密 切相关, 因此文中首先建立了决定其姿态和速度的控制模型, 并对飞行器面源热平衡方程进行建模研 究。然后, 应用 CFD (Computational Fluid Dynamics) 对飞行器的绝热壁温度场进行求解, 并给出了 机体绝热壁温度的高度简化算法。最后仿真了不同飞行器在不同波段、不同高度、不同速度下的红外 图像。
\end{abstract}

关键词: 红外图像；面源热平衡方程；CFD；绝热壁温度

中图分类号：TJ7；TP391 文献标志码：A 文章编号：1001-8891(2020)09-0863-10

\section{Simulation of Air Vehicle Motion Control Models and Infrared Images}

\author{
LIU Yongqiu ${ }^{1}$, JIN Zhongqing ${ }^{2}$ \\ (1. Guangdong University of Science and Technology, Dongguan 523083, China; \\ 2. Aviation University Air Force, Changchun 130000, China)
}

\begin{abstract}
The generation of infrared (IR) images of air vehicles in real time during flight is very important for research on the IR characteristics of air vehicles. The velocity, altitude, and attitude of air vehicles vary during flight. Moreover, the air vehicle body is affected by environmental and background radiation. Hence, it is very difficult to obtain an accurate temperature distribution of the air vehicle body. Because the temperature distribution is related to the state of motion, control models that determine the attitude and velocity are established, along with the surface heat balance equations. The adiabatic wall temperature distribution is solved by computational fluid dynamics, and simplified altitude algorithms are obtained. Finally, IR images of the air vehicle in different bands and at different altitudes and velocities are simulated.
\end{abstract}

Key words: IR image, surface heat balance equations, CFD, adiabatic wall temperature

\section{0 引言}

飞行器在高速飞行过程中其机体蒙皮表面与周 围的大气相互摩擦, 从而产生大量热量, 同时发动机 产生的热量也会通过热传导和热辐射传递到机体蒙 皮。因此在飞行过程中, 飞行器机体的温度明显高于 周围大气, 其红外特征较为明显。机体蒙皮各面源的 温度差异较大, 其机翼前缘、机头、座舱盖上部由于 与空气摩擦剧烈温度较高, 机身尾部贴近发动机喷口 处由于受发动机热效应的影响其蒙皮温度也较高, 机 体其他处温度较低, 因此各面源红外图像的灰度分布 也不一样。由于飞行器在飞行过程中其速度、高度、
姿态变化较大, 且其红外辐射易于受到环境、背景等 辐射的影响, 因此较难准确地仿真机体的温度场分 布。目前, 国内外研究飞行器点源红外特征的较多, 研究机体红外辐射特性的较少。刘娟 ${ }^{[1]}$ 研究了飞机的 红外辐射特性; 李建勋 ${ }^{[2]}$ 将机体的红外辐射分为机身 前部和尾部辐射两部分, 分别研究其红外特性; 黄鹤 松 ${ }^{[3]}$ 研究了自燃活性金属的热平衡方程, 并仿真了其 燃烧过程中的红外图像。

由于飞行器在飞行过程中其飞行姿态、速度、高 度以及各舵面的操纵量不断变化, 因此本文首先建立 飞行器的运动与控制模型, 得到其飞行过程中的各种 运动状态; 然后建立飞行器面源热平衡方程, 并应用 
计算流体力学 (Computational Fluid Dynamics, CFD) 对飞行器的绝热壁温度场进行了求解; 最后对飞行器 绝热壁温度场的计算结果进行高度简化, 并仿真了飞 行器在不同高度、速度、波段下的红外辐射图像。

由于飞行器发动机红外辐射特性方面的研究成 果较多, 因此本文采用文献[4-9]中的模型, 不再对其 进行单独建模。

\section{1 飞行器运动与控制模型}

飞行器红外辐射图像的仿真需要得到飞行器速 度、姿态、舵偏角等数据, 因此对飞行器的运动与控 制规律进行建模十分必要。飞行器在飞行中主要受到 的外力有: 气动力 $R$ 、自身重力 $G$ 以及发动机推力 $P$, 这些外力通常按不同的坐标系给出, 同时飞行器的运 动也需要在相应的坐标系内进行描述, 各坐标系之间 的关系详见参考文献[10]。

1) 飞行器质心运动动力学方程

$$
\left\{\begin{aligned}
m\left(\frac{\mathrm{d} V_{x}}{\mathrm{~d} t}+\omega_{y} V_{z}-\omega_{z} V_{y}\right)= & P \cos \varphi_{P}-X \cos \alpha \cos \beta+Y \sin \alpha \\
& -Z \cos \alpha \sin \beta-m_{\mathrm{g}} \sin \vartheta \\
m\left(\frac{\mathrm{d} V_{y}}{\mathrm{~d} t}+\omega_{z} V_{x}-\omega_{x} V_{z}\right)= & P \sin \varphi_{P}+X \sin \alpha \cos \beta+Y \cos \alpha \\
& +Z \sin \alpha \sin \beta-m_{\mathrm{g}} \cos \gamma \cos \vartheta \\
m\left(\frac{\mathrm{d} V_{z}}{\mathrm{~d} t}+\omega_{x} V_{y}-\omega_{y} V_{x}\right)= & -X \sin \beta+Z \cos \beta+m_{\mathrm{g}} \sin \gamma \cos \vartheta
\end{aligned}\right.
$$

式中: $V_{x} 、 V_{y} 、 V_{z} 、 \omega_{x} 、 \omega_{y} 、 \omega_{z}$ 分别为飞行器三轴速 度、角速度分量; $P$ 为发动机的推力; $m_{\mathrm{g}}$ 为自身重力; $\alpha 、 \beta 、 \vartheta 、 \gamma 、 \varphi_{P}$ 分别为迎角、侧滑角、俯仰角、滚 转角以及发动机安装角; $X 、 Y 、 Z$ 分别为气动阻力、 气动升力以及侧力; $t$ 为时间。

2) 飞行器绕质心转动动力学方程

$$
\left\{\begin{array}{l}
I_{x} \frac{\mathrm{d} \omega_{x}}{\mathrm{~d} t}-\left(I_{y}-I_{z}\right) \omega_{y} \omega_{z}-I_{x y}\left(\frac{\mathrm{d} \omega_{y}}{\mathrm{~d} t}-\omega_{z} \omega_{x}\right)=\sum M_{x} \\
I_{y} \frac{\mathrm{d} \omega_{y}}{\mathrm{~d} t}-\left(I_{z}-I_{x}\right) \omega_{z} \omega_{x}-I_{x y}\left(\frac{\mathrm{d} \omega_{x}}{\mathrm{~d} t}+\omega_{y} \omega_{z}\right)=\sum M_{y} \\
I_{z} \frac{\mathrm{d} \omega_{z}}{\mathrm{~d} t}-\left(I_{x}-I_{y}\right) \omega_{x} \omega_{y}-I_{x y}\left(\omega_{x}^{2}-\omega_{y}^{2}\right)=\sum M_{z}
\end{array}\right.
$$

式中: $\sum M_{x} 、 \sum M_{y} 、 \sum M_{z}$ 为飞行器的合外力矩; $I_{x} 、 I_{y}$ 、 $I_{z}$ 为飞行器的三轴惯性矩; $I_{x y} 、 I_{y z} 、 I_{z x}$ 为飞行器的惯 性积, 其中 $I_{y z}=0, I_{z x}=0$ 。

3) 飞行器绕质心转动运动学方程

$$
\left\{\begin{array}{l}
\frac{\mathrm{d} \gamma}{\mathrm{d} t}=\omega_{x}-\tan \vartheta\left(\omega_{y} \cos \gamma-\omega_{z} \sin \gamma\right) \\
\frac{\mathrm{d} \psi}{\mathrm{d} t}=\frac{1}{\cos \vartheta}\left(\omega_{y} \cos \gamma-\omega_{z} \sin \gamma\right) \\
\frac{\mathrm{d} \vartheta}{\mathrm{d} t}=\omega_{y} \sin \gamma+\omega_{z} \cos \gamma
\end{array}\right.
$$

联立公式(1) (3), 在知道了飞行器的空气动力特 性、发动机推力特性和发动机安装角 $\varphi_{P}$ 、初始飞行状 态和各种姿态角后, 通过数值积分, 即可解得飞行器 在运动过程中的速度和姿态。

\section{2 飞行器机体热平衡方程}

飞行器在高速飞行过程中机体与空气摩擦, 机体 蒙皮温度上升, 其辐射亮度值主要取决于机体蒙皮表 面的温度场及机体蒙皮发射率。机体蒙皮发射率一般 可当作固定值处理, 因此求解机体的红外辐射亮度关 键在于准确地求解机体表面的温度场。

机体的表面温度与机体表面换热量有以下关系:

$$
c \frac{\mathrm{d} T}{\mathrm{~d} t}=\Delta Q
$$

式中: $\Delta Q$ 为单位时间内单位面积的机体蒙皮上的热 量变化值; $T$ 为机体蒙皮温度; $c$ 为比热容, 则有:

$$
\Delta Q=Q_{\text {in }}+Q_{\text {rad }}+Q_{\text {flow }}+Q_{\text {env }}
$$

式中: $Q_{\text {in }}$ 为蒙皮之间热传导、热辐射产生的能量; $Q_{\mathrm{rad}}$ 为蒙皮向外界辐射的能量; $Q_{\text {flow }}$ 为蒙皮与周围大 气之间的换热量; $Q_{\mathrm{env}}$ 为蒙皮接受到周围环境辐射的 能量。

\section{1 蒙皮之间热传导、热辐射产生的能量}

蒙皮之间热传导、热辐射的能量主要发生在发动 机舱, 飞行器在飞行过程中, 由于发动机燃烧室内猛 烈持续的燃烧反应, 发动机的各个部件都会产生大量 的热量, 这些热量大部分通过发动机的冷却系统进行 冷却, 少部分热量通过热传导和热辐射的方式传递到 相邻的其他蒙皮上。蒙皮之间热传导、热辐射的能量 可以由下式得到:

$$
Q_{\text {in }}=\alpha_{\text {sur }} \varepsilon_{\text {in }} \sigma T_{\text {in }}^{4} F_{\text {in-sur }} A_{\mathrm{o}}+\frac{\lambda_{\mathrm{c}}}{l_{\text {in-sur }}}\left(T_{\text {in }}-T_{\text {sur }}\right)
$$

式中: $T_{\text {in }}$ 为发动机的简化温度; $\varepsilon_{\text {in }}$ 为发动机的发射 率; $\alpha_{\text {sur }}$ 为蒙皮表面的吸收率; $F_{\text {in-sur }}$ 为辐射角系数; $\lambda_{\mathrm{c}}$ 为热传导系数; $l_{\text {in-sur }}$ 为传导距离; $T_{\mathrm{sur}}$ 为蒙皮表面 温度; $\sigma$ 为斯忒藩-玻尔兹曼常数, 其值为 $5.67 \times 10^{-8}$ $\mathrm{W} \cdot \mathrm{m}^{-2} \cdot \mathrm{K}^{-4}$ 。

\section{2 机体表面向外辐射热量}

由玻尔兹曼定律，黑体的辐射出射度为: 


$$
M=\sigma T^{4}
$$

将机体蒙皮简化为发射率 $\varepsilon_{\text {sur }}$ 的灰体, 则单位时 间内机体蒙皮自身辐射的热量为:

$$
Q_{\mathrm{rad}}=-\varepsilon_{\text {sur }} \sigma T_{\text {sur }}{ }^{4}
$$

\section{3 环境辐射热量}

环境辐射主要包括大气辐射、海洋辐射、地面辐 射、云层辐射、太阳辐射等, 由于飞行器在高空中飞 行, 地面辐射对其影响不大, 本文只考虑对机体蒙皮 影响最大的大气辐射和太阳辐射 ${ }^{[11-12]}$ 。环境辐射可由 下式得到:

$$
Q_{\text {env }}=Q_{\text {sun }}+Q_{\text {air }}=\alpha_{\text {sur }} E_{\text {sun }} \tau_{\mathrm{h}} \cos \theta_{\mathrm{s}} \delta_{\theta_{\mathrm{s}}}+\alpha_{\text {sur }} \sigma T_{\infty}^{4}
$$

式中: $Q_{\text {sun }}$ 为太阳辐射能量; $Q_{\text {air }}$ 为大气辐射能量; $\tau_{\mathrm{h}}$ 为传输路径上的大气透过率; $\theta_{\mathrm{s}}$ 为太阳直射光线与蒙 皮面元法线方向之间的夹角; $E_{\mathrm{sun}}=1353 \mathrm{~W} / \mathrm{m}^{2}$ 为太阳 的有效辐照度; $\delta_{\theta_{\mathrm{s}}}$ 为蒙皮外表面的标识量, 当蒙皮 面元与太阳光之间没有遮挡时, $\delta_{\theta_{\mathrm{s}}}=1$, 否则 $\delta_{\theta_{\mathrm{s}}}=0$ 。

\section{4 机体蒙皮与周围大气的换热量}

飞行器在空中飞行过程中, 其机体蒙皮表面与周 围大气摩擦, 摩擦作用使周围气体温度上升。同时, 在飞行过程中, 飞行器蒙皮表面压缩前方气体, 使得 气体温度进一步升高。在飞行器的飞行过程中, 机体 的附面层气体温度较高, 则机体蒙皮与附面层气体的 换热量为:

$$
Q_{\text {flow }}=\alpha_{\text {flow }}\left(T_{\text {flow }}-T_{\text {sur }}\right)
$$

式中: $T_{\text {flow }}$ 为附面层气体温度; $\alpha_{\text {flow }}$ 为换热系数。换 热系数 $\alpha_{\text {flow }}$ 主要由空气导热系数 $\lambda_{\mathrm{a}}$; 机体特征长度 $x$ 以及雷诺数 $\operatorname{Re}_{x}$ 确定, 其值为:

$$
\alpha_{\text {flow }}=\frac{0.332 \operatorname{Re}_{x}^{1 / 2} \operatorname{Pr}^{1 / 3} \lambda_{\mathrm{a}}}{x}
$$

式中: $\mathrm{Re}_{x}$ 为雷诺数; $\operatorname{Pr}$ 为普朗特数, 空气的 Pr 数一 般取 0.72 。附面层气体的温度 $T_{\text {flow }}$ 既受飞行器外形的 影响, 又与飞行器的飞行速度、高度、大气温度、大 气密度等有关, 无法精确获得。文中采用 CFD 计算 飞行器机体蒙皮表面的绝热壁温度, 然后用绝热壁温 度来近似代表 $T_{\text {flow }}$, 求解过程中设置飞行器的机体表 面为绝热壁 ${ }^{[13]}$ 。

\section{3 机体绝热壁温度计算}

\section{1 网格刨分}

首先建立飞行器的三维几何模型。为了保证模型 的精确性, 本文采用 CATIA 三维建模软件对飞行器 的三维模型进行建模, 以 F-22 为例, 建立的三维几 何模型如图 1 所示。

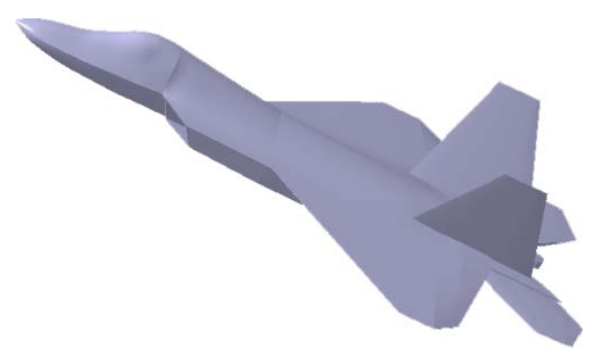

图 1 F-22 三维模型图

Fig.1 Three-dimensional model diagram of F-22

利用 ICEM 软件对计算域及机体表面进行网格划 分。本文以 F-22 为例, 设计算区域为 $210 \mathrm{~m} \times 70 \mathrm{~m} \times 70$ $\mathrm{m}$ 的长方体, 飞行器中心与计算域的中心相重合, 如 图 2 所示。边界层网格第一层厚度为 $10^{-3} \mathrm{~m}$, 边界层 一共 12 层, 采用四面体网格, 如图 3 4 所示。

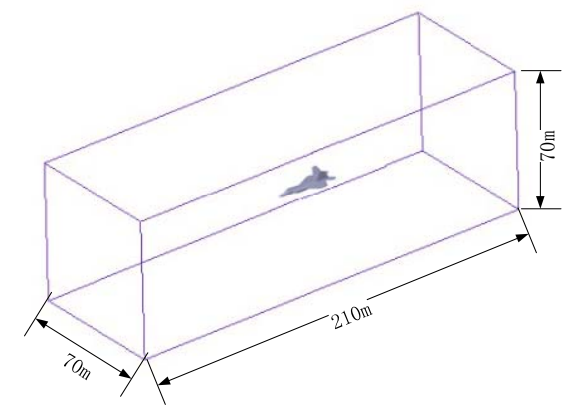

图 2 计算域示意图

Fig.2 Schematic diagram of computational domain

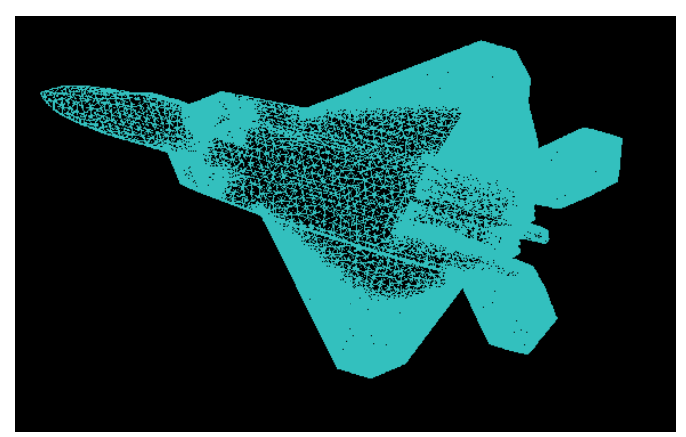

图 3 F-22 机体表面网格图

Fig.3 Grid diagram of F-22

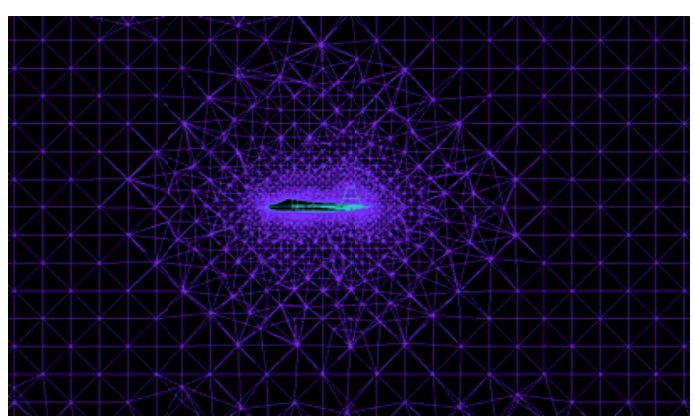

图 4 计算域网格图

Fig.4 Grid diagram of computational domain 


\subsection{CFD 流场计算}

控制方程选用 Navier-Stokes 方程。采用有限体积 的空间离散算法和空间二阶精度的线性插值算法, 时 间离散采用二阶精度的后向差分算法，计算模型选用 SST 湍流模型 ${ }^{[10]}$ 。计算域外边界设置为压力远场边 界, 机体表面设置为无滑移绝热壁面, 进气道入口设 置为质量入口, 质量流量由发动机气体流量数据库决 定, 尾喷口设置为压力出口, 认为喷口气体处于完全 膨胀状态，则喷口静压与外界大气压力相同。

设置的初始条件为高度 $11 \mathrm{~km}$, 压力 $P=22700$ $\mathrm{Pa}$, 大气温度 $T=216.774 \mathrm{~K}$, 飞行器飞行马赫数 $\mathrm{Ma}$ $=1.6$ 。则 F-22 飞机绝热壁温度分布计算结果如图 5 所示。
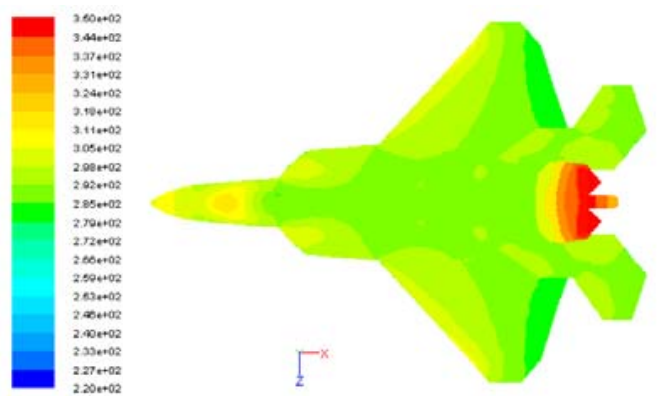

图 5 F-22 飞机绝热壁温度分布图

Fig.5 Diathermal wall temperature distribution diagram of F-22

飞行器在稳定飞行过程中，机体蒙皮各面源温度 不变, 则公式(5)中的 $\Delta Q=0$ 。因此, 在得到飞行器各 面源的绝热壁温度 $T_{\text {flow }}$ 后, 联立公式(5) (11), 便可 以求解出飞行器机体蒙皮的表面温度 $T_{\text {sur。 }}$ 。

\section{3 高度简化算法}

由于飞行器的飞行高度通常在 $0 \sim 20 \mathrm{~km}$ 内, 飞 行马赫数在 $0 \sim 2$ 范围内变化, 飞行过程中由于舵面 的操纵, 迎角和侧滑角也都在变化。因此要计算飞行 器在运动过程中的红外辐射图像, 需要对飞行器在各 种条件下的绝热壁温度进行计算, 计算量十分庞大, 需要对 CFD 计算结果进行简化。

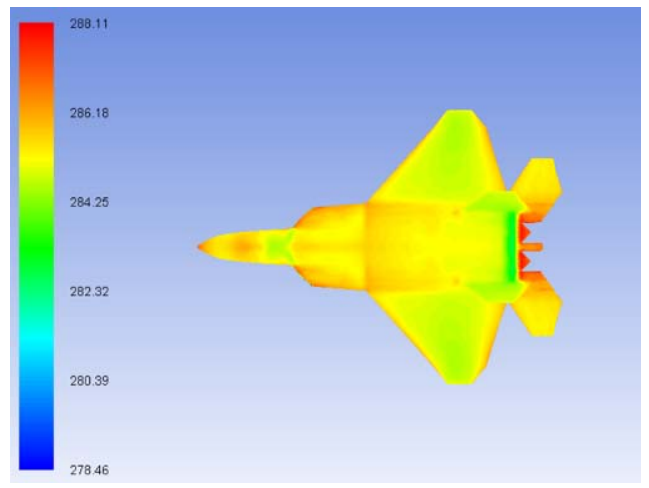

(a) $h=3 \mathrm{~km}, k=0.98-1.01$
当飞行器飞行速度增加时, 其机翼表面上的雷诺 数也跟着增加, 而当雷诺数增加到一定数值后, 机体 表面的附面层就会出现转戻现象, 从而出现紊流, 使 得机体表面的温度分布差异很大。因此飞行器蒙皮的 绝热壁温度分布随其飞行速度的变化关系较为复杂、 无章，无法对其进行简化。而飞行高度主要决定着周 围大气的环境温度, 对飞行器机体的温度分布影响一 般较小, 因此本节研究飞行器机体绝热壁温度的高度 简化算法。

飞行器机体的驻点温度可由下式得到:

$$
T_{\mathrm{b}}=T_{0}\left(1+r \frac{\gamma-1}{2} \mathrm{Ma}^{2}\right)
$$

式中: $T_{0}$ 为大气环境温度 $; r$ 为恢复温度系数 (层流 取值一般为 0.82 , 湍流为 0.87 ）； $\gamma$ 为大气绝热指数, $\gamma=1.4 ; T_{\mathrm{b}}$ 为驻点温度。设机体蒙皮的绝热温度与驻 点温度比为 $k$, 即:

$$
T_{\text {flow }}=k T_{\mathrm{b}}
$$

式中: $k$ 表示机体蒙皮各面源与驻点温度的比值, 也 表示了机体的温度分布情况。

设 F-22 飞机飞行马赫数为 0.6 , 则图 6 为其在不 同高度下机体绝热壁温度场的 CFD 计算结果。

由图 6 可知, 当飞行器的飞行马赫数相同时, 不 同高度下飞行器的绝热壁温度分布基本不变。设蒙皮 面源 $i$ 在 $h \mathrm{~km}$ 高度时绝热壁温度为 $T_{h, i}, T_{h}$ 为飞行器 在 $h \mathrm{~km}$ 高度时的驻点温度, 则蒙皮面源 $i$ 的简化结果 为:

$$
\begin{gathered}
T_{h, i}^{\prime}=\left(T_{h} / T_{0}\right) T_{10, i} \\
\varepsilon_{h, i}=\left|T_{h, i}^{\prime}-T_{h, i}\right|
\end{gathered}
$$

式中: $T_{h, i}{ }^{\prime}$ 为面源 $i$ 的绝热壁温度简化计算结果; $\varepsilon_{h, i}$ 为采用简化算法后的面源 $i$ 的绝对误差。采用简化算 法得到的误差对比分布图如图 7 和图 8 所示。

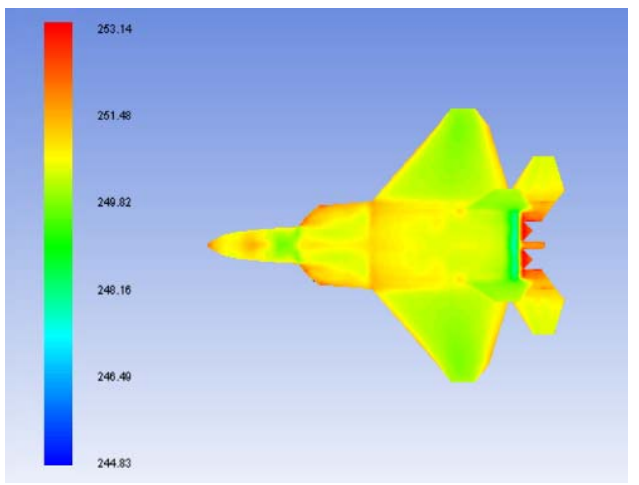

(b) $h=8 \mathrm{~km}, k=0.98-1.01$ 


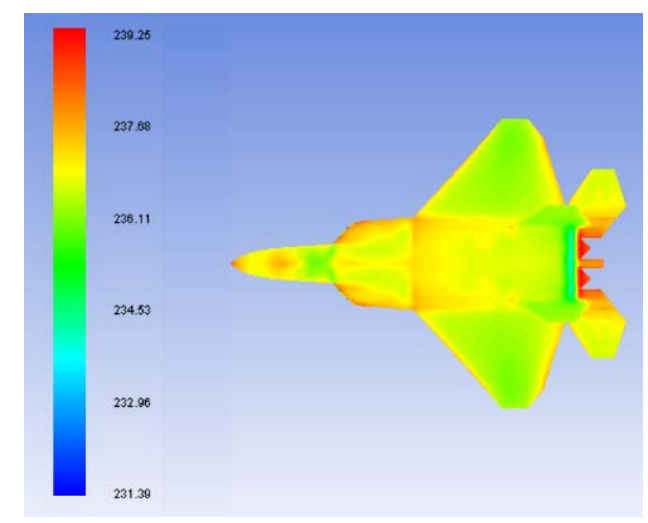

(c) $h=10 \mathrm{~km}, k=0.98-1.01$

图 6 不同高度下的机体绝热壁温度分布

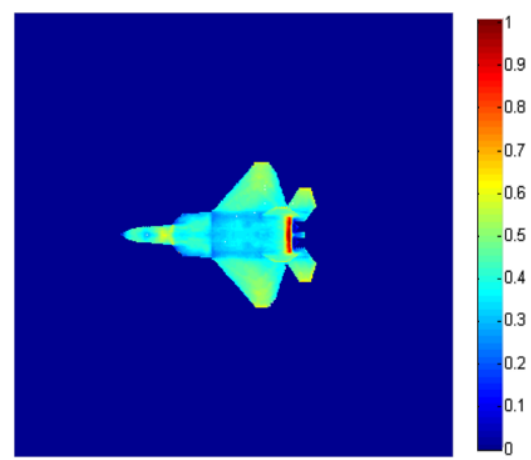

(a) $h=3 \mathrm{~km}$ ，机体上表面

(a) Upper surface of air vehicle at $h=3 \mathrm{~km}$

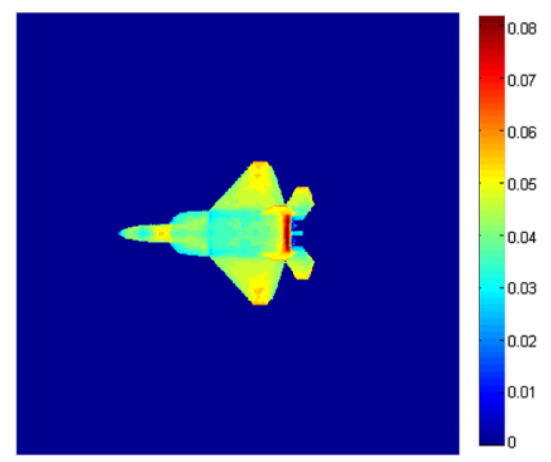

(c) $h=8 \mathrm{~km}$, 机体上表面

(c) Upper surface of air vehicle at $h=8 \mathrm{~km}$

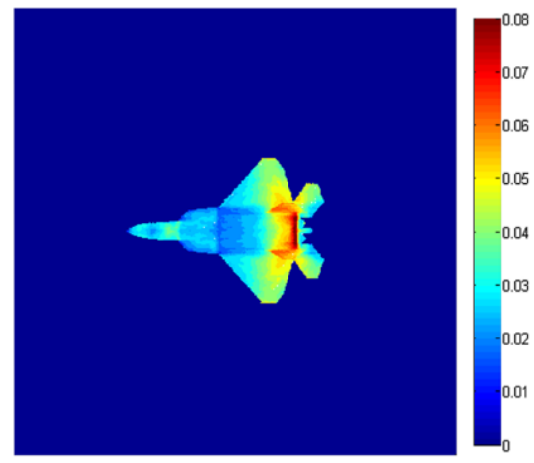

(e) $h=13 \mathrm{~km} ，$ 机体上表面

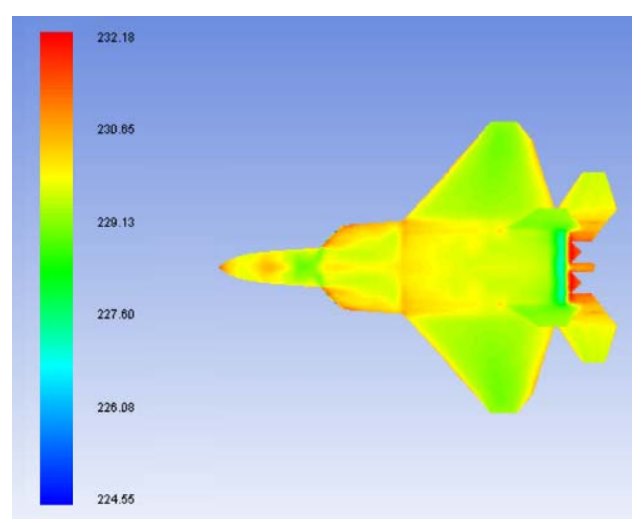

(d) $h=13 \mathrm{~km}, k=0.98-1.01$

Fig.6 Diathermal wall temperature distribution at different altitudes

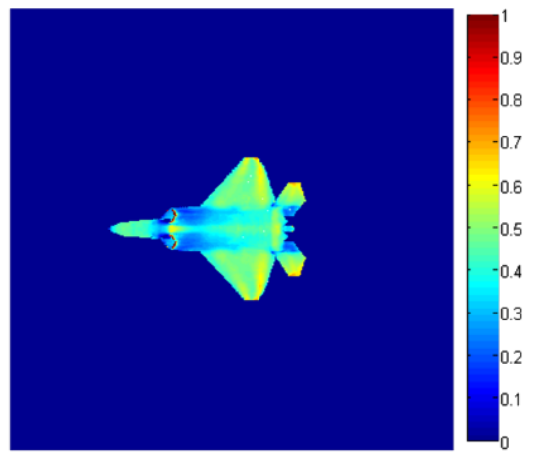

(b) $h=3 \mathrm{~km}$, 机体下表面

(b) Lower surface of air vehicle $h=3 \mathrm{~km}$

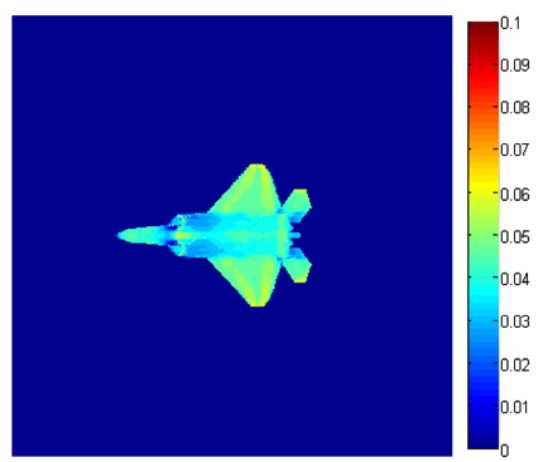

(d) $h=8 \mathrm{~km}$ ，机体下表面

(d) Lower surface of air vehicle at $h=8 \mathrm{~km}$

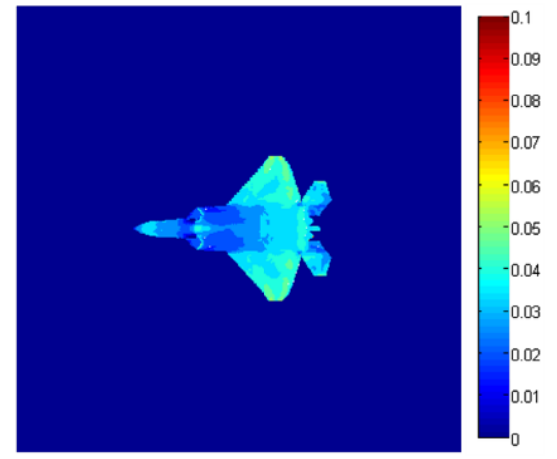

(f) $h=13 \mathrm{~km}$ ，机体下表面

(e) Upper surface of air vehicle at $h=13 \mathrm{~km} \quad$ (f) Lower surface of air vehicle at $h=13 \mathrm{~km}$

图 7 各高度下 $\varepsilon_{h, i}$ 的分布图 $(\mathrm{Ma}=0.6)$

Fig.7 $\varepsilon_{h, i}$ distribution diagram at different altitudes $(\mathrm{Ma}=0.6)$ 


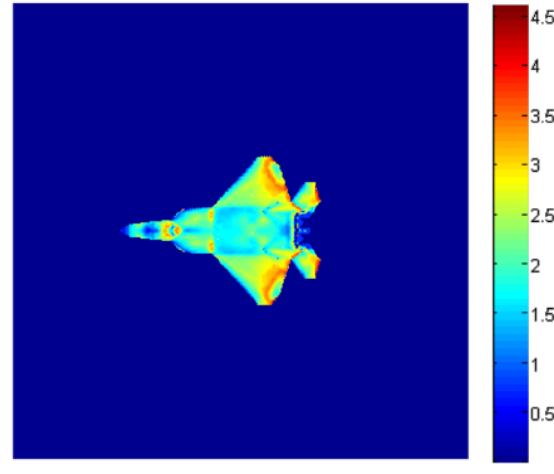

(a) $h=3 \mathrm{~km} ，$ 机体上表面

(a) Upper surface of air vehicle at $h=3 \mathrm{~km}$

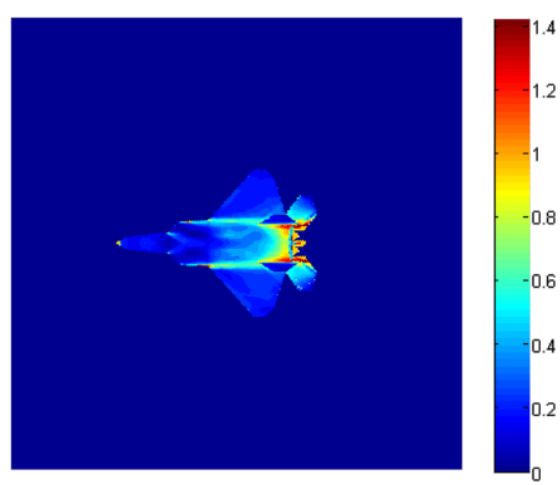

(c) $h=8 \mathrm{~km}$ ，机体上表面

(c) Upper surface of air vehicle at $h=8 \mathrm{~km}$

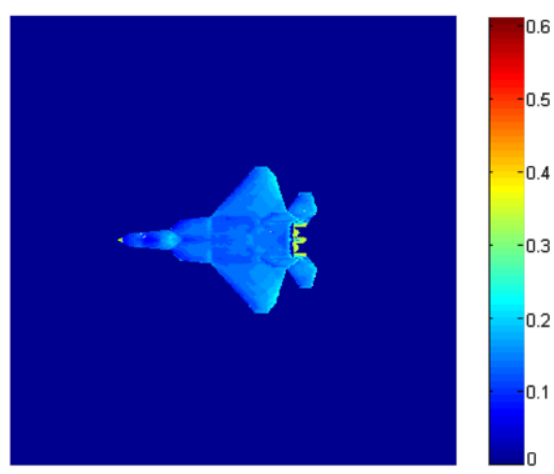

(e) $h=13 \mathrm{~km}$ ，机体上表面

(e) Upper surface of air vehicle at $h=13 \mathrm{~km}$

图 8 各高度下 $\varepsilon_{h, i}$ 的分布图 $(\mathrm{Ma}=1.5) \quad$ Fig.8

由图 7 和图 8 可知, 当飞行马赫数为 0.6 时, 绝 对误差 $\varepsilon_{h, i}$ 的值全部小于 $1 \mathrm{~K}$; 当飞行马赫数为 1.5 时, 绝对误差 $\varepsilon_{h, i}$ 的值全部小于 $4.5 \mathrm{~K}$, 且只有当高度为 3 $\mathrm{km}$ 时, 少部分面源的绝对误差 $\varepsilon_{h, i}$ 值大于 $3 \mathrm{~K}$, 其余 条件下全部小于 $1.8 \mathrm{~K}$ 。由图 9 可知, 飞行高度在 $10 \mathrm{~km}$ 附近误差最小, 飞行高度在海平面和 $20 \mathrm{~km}$ 时, 误差 最大, 但整体误差仍较小。因此采用公式(14)的简化 算法计算得到的绝热壁温度结果较为准确, 能够满足 计算精度的要求。

\section{4 机体红外辐射图像仿真}

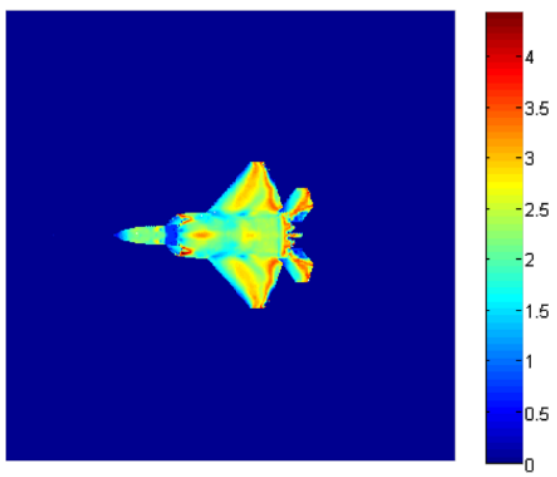

(b) $h=3 \mathrm{~km}$, 机体下表面

(b) Lower surface of air vehicle at $h=3 \mathrm{~km}$

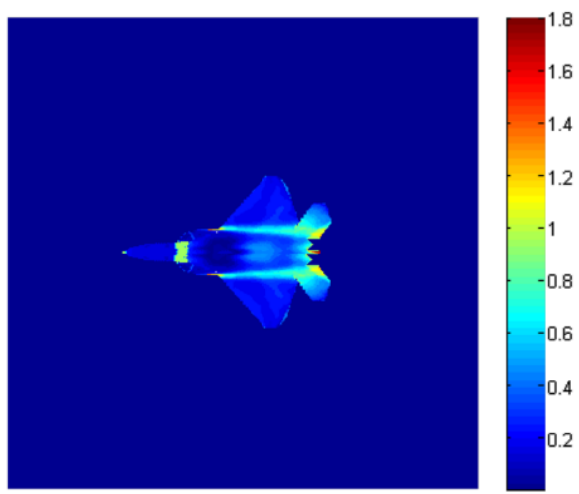

(b) $h=8 \mathrm{~km}$, 机体下表面

(d) Lower surface of air vehicle at $h=8 \mathrm{~km}$

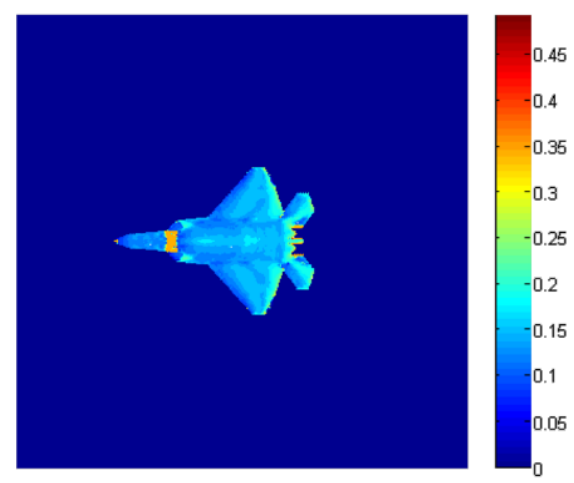

(f) $h=13 \mathrm{~km}$, 机体下表面

(f) Lower surface of air vehicle at $h=13 \mathrm{~km}$

$\varepsilon_{h, i}$ distribution diagram at different altitudes $(\mathrm{Ma}=1.5)$

令 $\Delta Q=0$, 则由公式(5)及飞行器的绝热壁温度 CFD 计算结果, 可以求得飞行器蒙皮任意面源 $i$ 的温 度, 从而得到飞行器在飞行过程中的温度分布结果。 本节应用 VC+ + 语言, 借助 OpenGL 绘图工具绘制 飞行器的红外辐射图像。

设 F-22 飞机水平飞行, 飞行马赫数为 0.6 。飞行 器平尾舵偏角 $\delta_{z b}=5^{\circ}$, 垂尾和副翼的舵偏角 $\delta_{y b}=0^{\circ}$ 、 $\delta_{x b}=0^{\circ}$, 则其在不同高度下的红外图像如图 10 所示。

设 F-22 飞机水平飞行, 飞行马赫数为 1.0 。飞行 器平尾舵偏角 $\delta_{z b}=3^{\circ}$, 垂尾和副翼的舵偏角 $\delta_{y b}=0^{\circ}$ 、 
$\delta_{x b}=0^{\circ}$, 则其在不同高度下的红外图像如图 11 所示。

设 F-16 飞机水平飞行, 飞行马赫数为 0.6 时, 平 尾舵偏角 $\delta_{z b}=7^{\circ}$, 垂尾和副翼的舵偏角 $\delta_{y b}=0^{\circ} 、 \delta_{x b}$ $=0^{\circ}$; 飞行马赫数为 1.0 时, 平尾舵偏角 $\delta_{z b}=4^{\circ}$, 垂 尾和副翼的舵偏角 $\delta_{y b}=0^{\circ} 、 \delta_{x b}=0^{\circ}$, 则其在不同高度 下的红外图像仿真结果如图 12 和图 13 所示。

由图 10 图 13 的仿真结果可知, 飞行器红外图

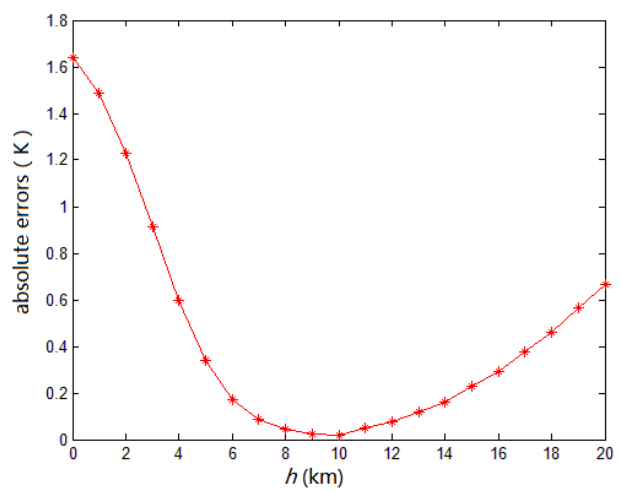

(a) $\mathrm{Ma}=0.6$

图 9 绝对误差最大值随高度变化的曲线

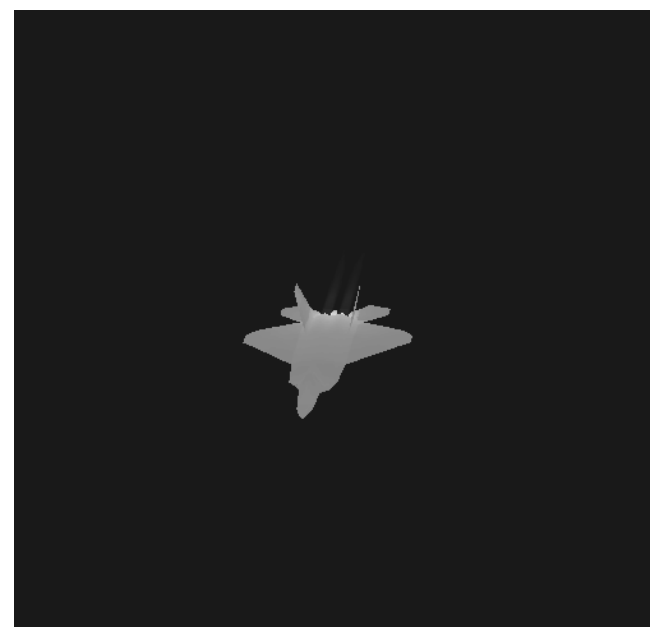

(a) $h=1 \mathrm{~km}, 8-12 \mu \mathrm{m}$

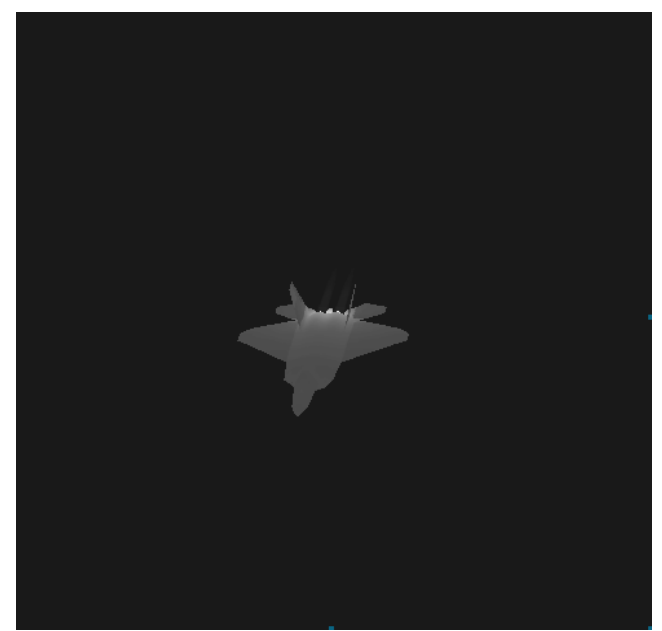

(c) $h=8 \mathrm{~km}, 8-12 \mu \mathrm{m}$

图 $10 \mathrm{~F}-22$ 红外辐射图像 $(\mathrm{Ma}=0.6)$
像的亮度随着飞行高度的上升而下降, 随着飞行速度 的增加而增加, 同时 8 12 $\mu \mathrm{m}$ 波段的图像亮度明显 高于 3 5 $\mu \mathrm{m}$ 波段。亮度越高的点表示此处的红外辐 射亮度越强, 相应的温度也越高。对比相同条件下的 仿真结果可知, F-22 飞机的红外辐射强度略大于 F16, 这是由于文中仿真未考虑 F-22 飞机隐身涂层, 且 F-22 飞机机身蒙皮面积大于 F-16 所致。

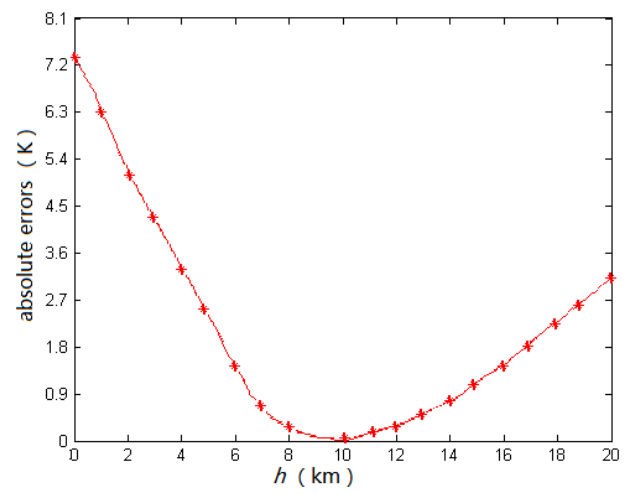

(b) $\mathrm{Ma}=1.5$

Fig.9 Maximal absolute error vary with altitude

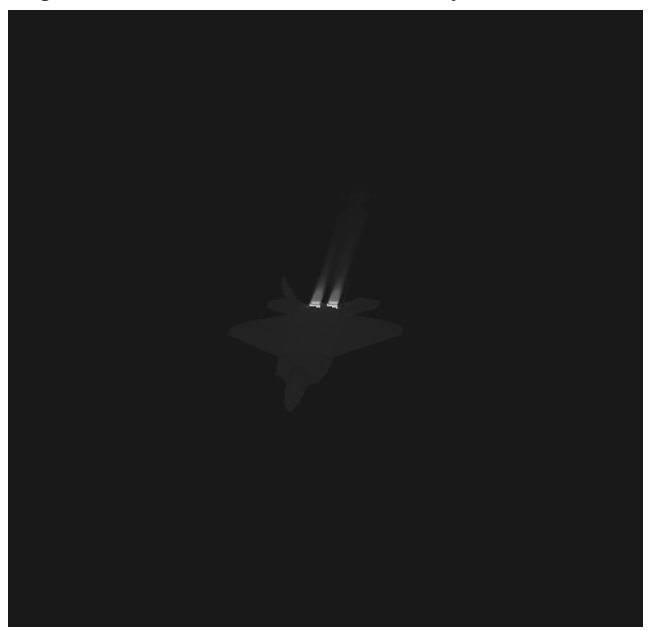

(b) $h=1 \mathrm{~km}, 3-5 \mu \mathrm{m}$

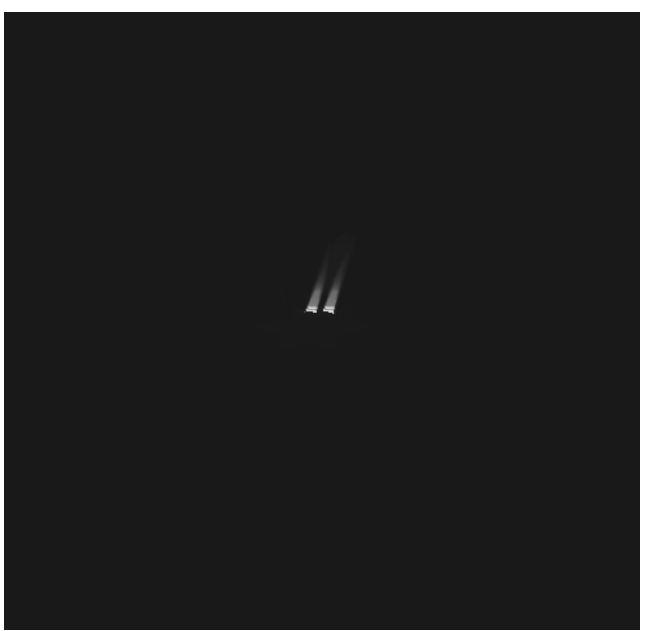

(d) $h=8 \mathrm{~km}, 3-5 \mu \mathrm{m}$

Fig.10 IR radiation image of $\mathrm{F}-22$ at $0.6 \mathrm{Ma}$ 

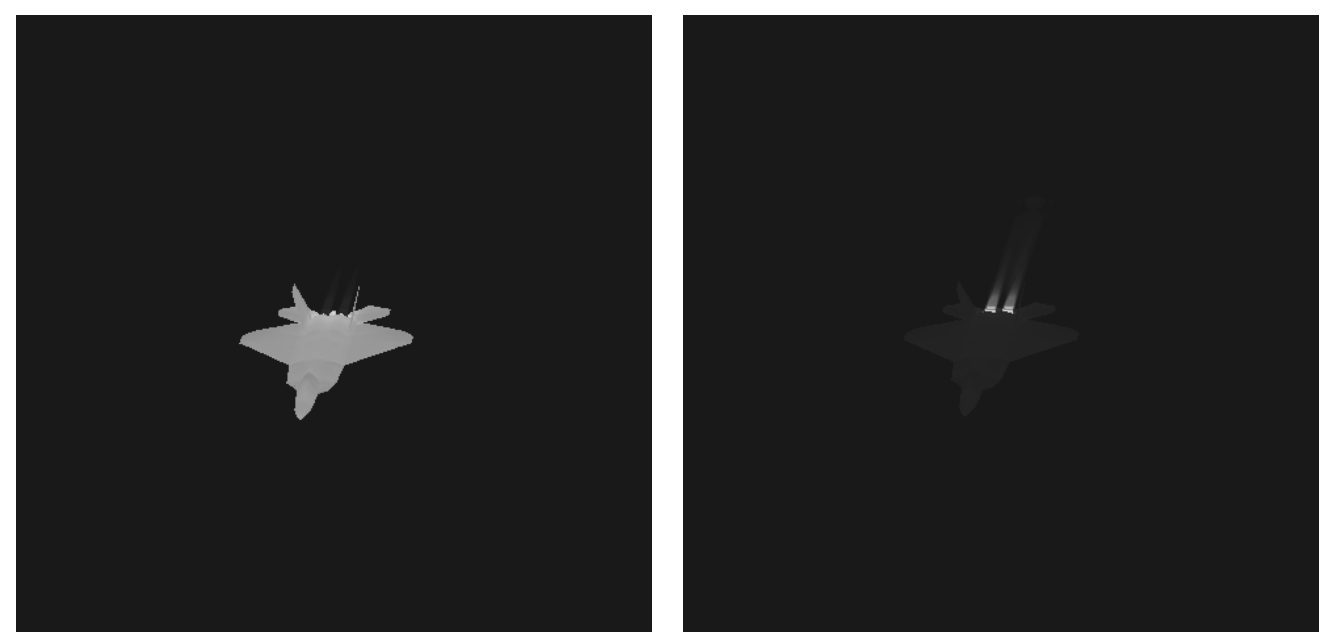

(a) $h=1 \mathrm{~km}, 8-12 \mu \mathrm{m}$

(b) $h=1 \mathrm{~km}, 3-5 \mu \mathrm{m}$
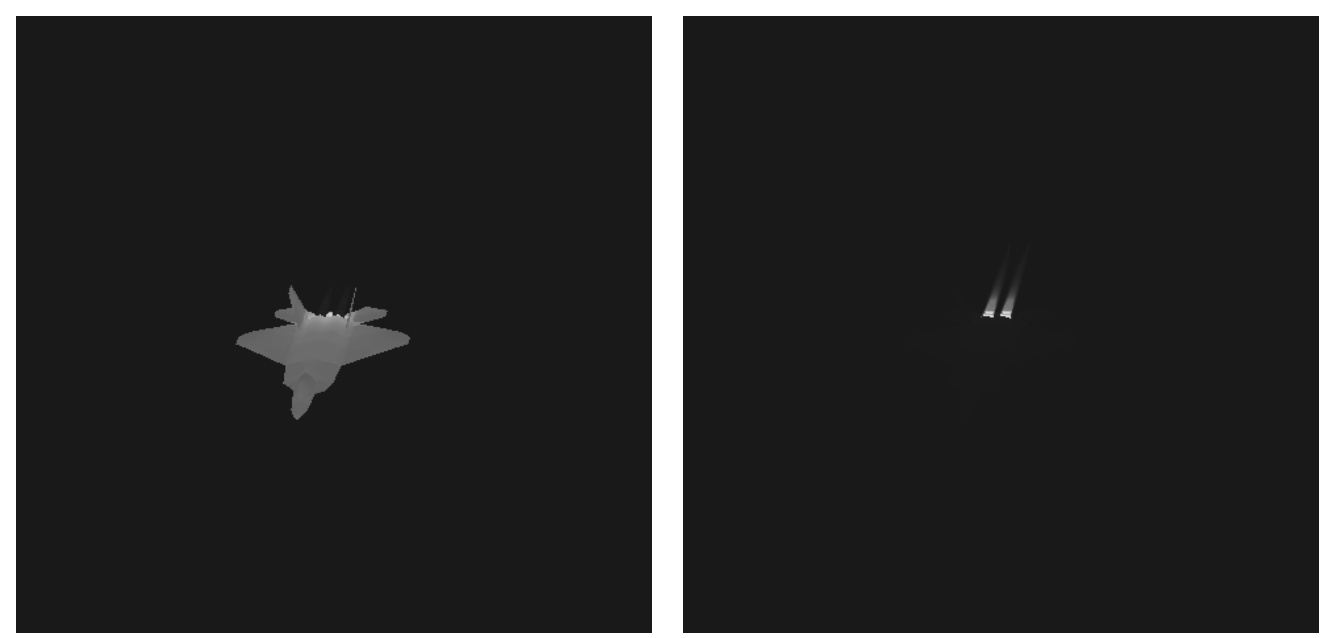

(c) $h=8 \mathrm{~km}, 8-12 \mu \mathrm{m}$

(d) $h=8 \mathrm{~km}, 3-5 \mu \mathrm{m}$

图 11 F-22 红外辐射图像 $(\mathrm{Ma}=1.0)$

Fig.11 IR radiation image of F-22 at $1.0 \mathrm{Ma}$
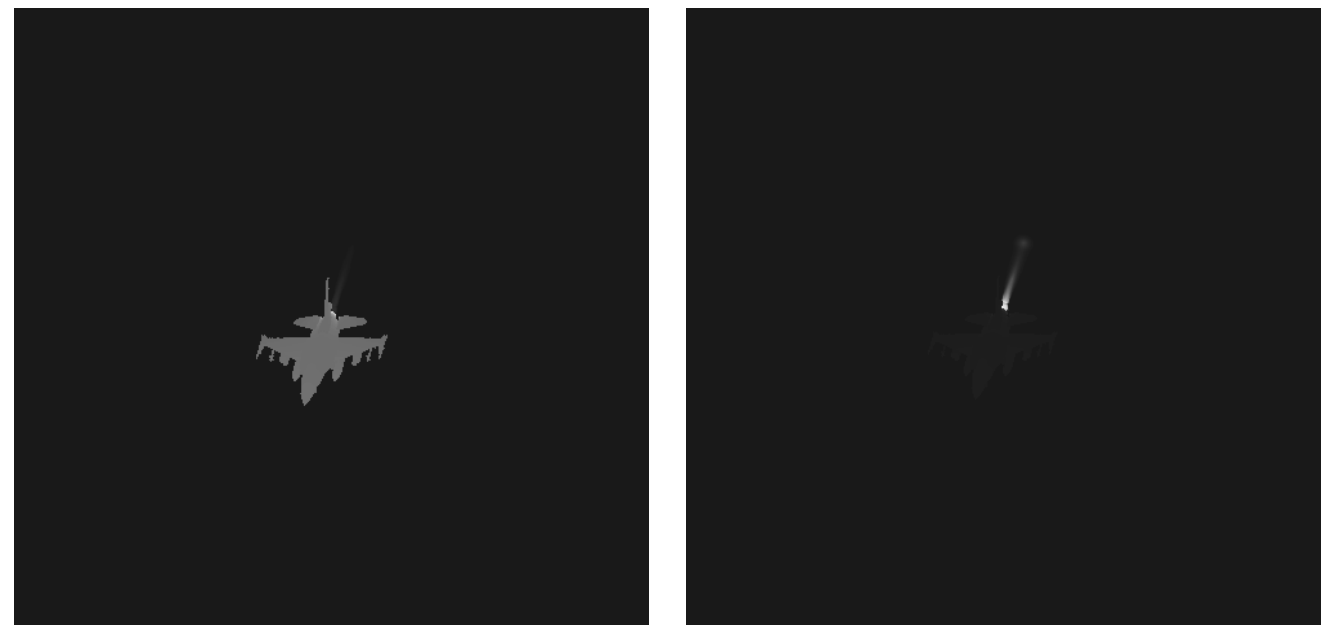

(a) $h=1 \mathrm{~km}, 8-12 \mu \mathrm{m}$

(b) $h=1 \mathrm{~km}, 3-5 \mu \mathrm{m}$ 

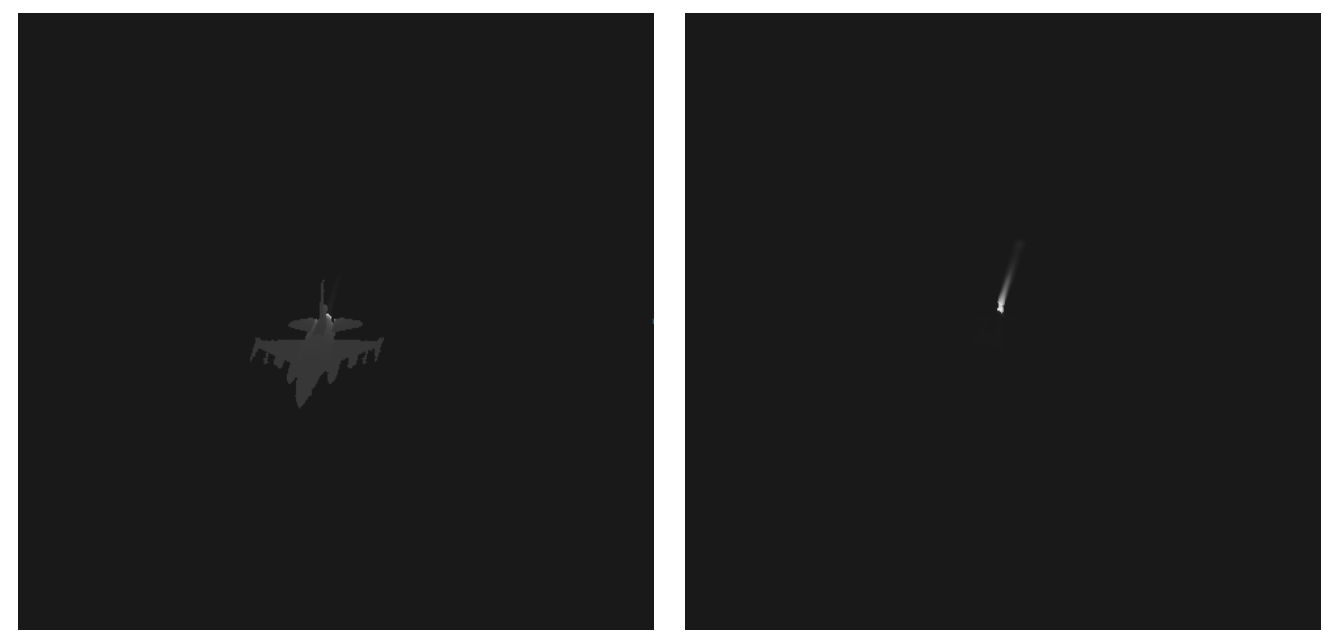

(c) $h=8 \mathrm{~km}, 8-12 \mu \mathrm{m}$

(d) $h=8 \mathrm{~km}, 3-5 \mu \mathrm{m}$

图 12 F-16 红外辐射图像 $(\mathrm{Ma}=0.6)$

Fig.12 IR radiation image of F-16 at $0.6 \mathrm{Ma}$
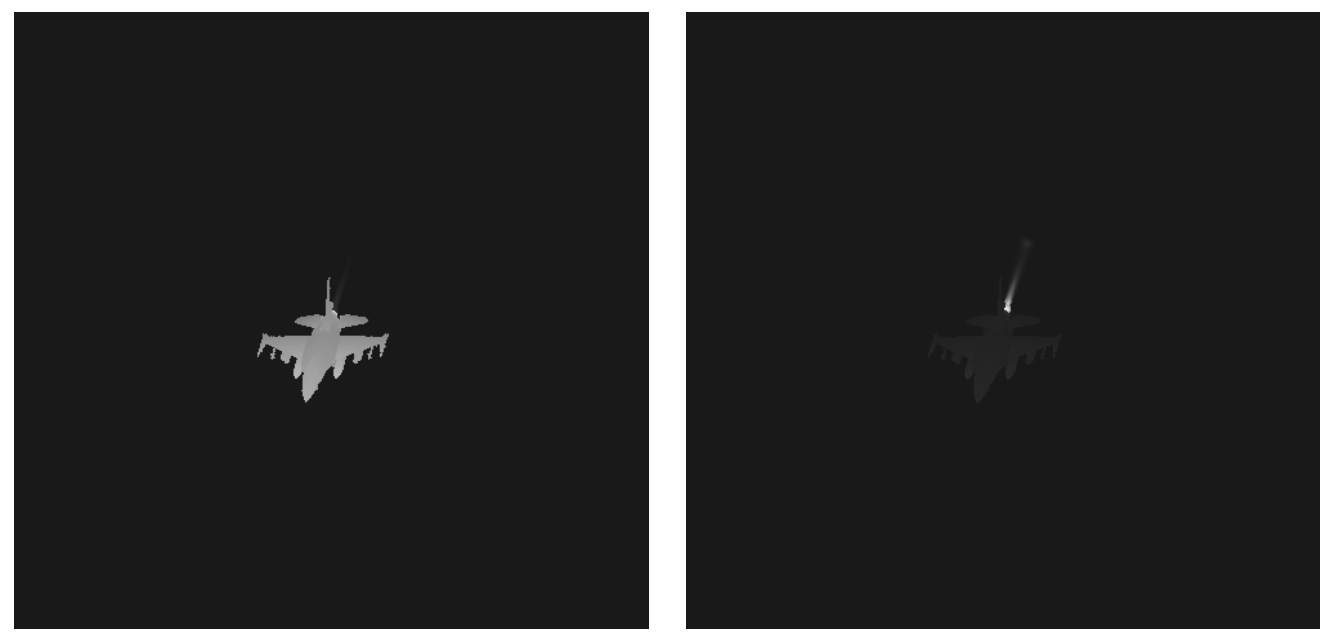

(a) $h=1 \mathrm{~km}, 8-12 \mu \mathrm{m}$

(b) $h=1 \mathrm{~km}, 3-5 \mu \mathrm{m}$
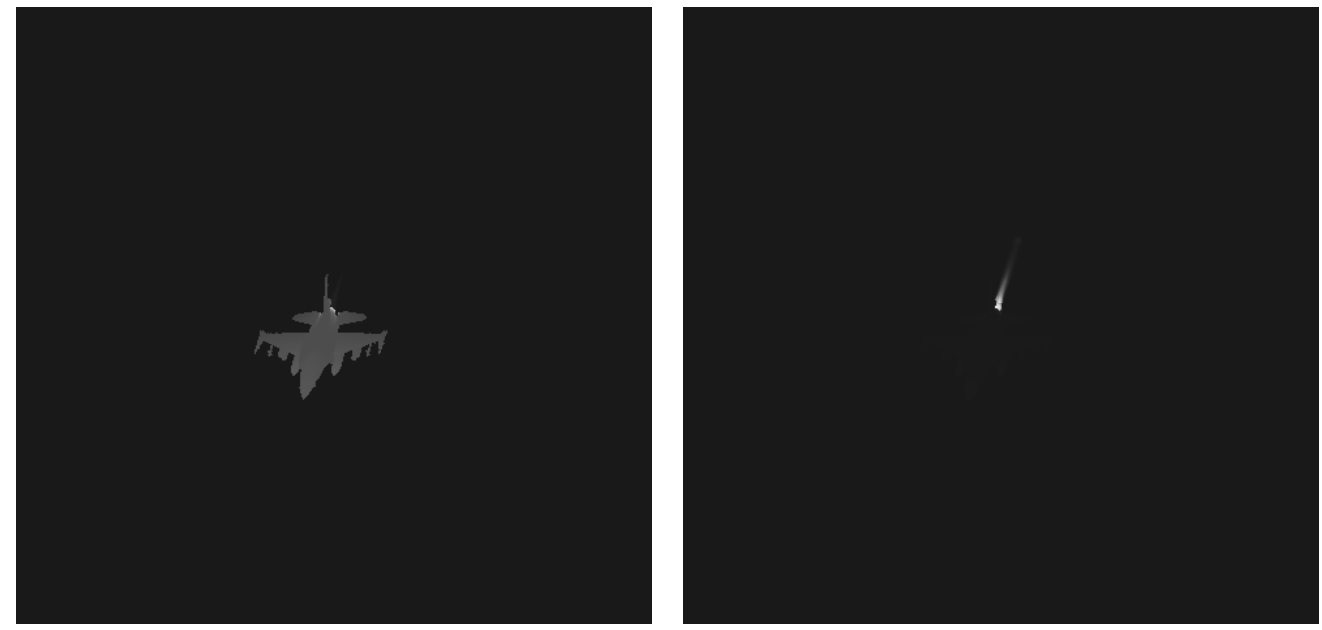

(c) $h=8 \mathrm{~km}, 8-12 \mu \mathrm{m}$

(d) $h=8 \mathrm{~km}, 3-5 \mu \mathrm{m}$

图 $13 \mathrm{~F}-16$ 红外辐射图像 $(\mathrm{Ma}=1.0)$

Fig.13 IR radiation image of F-16 at $1.0 \mathrm{Ma}$ 


\section{5 结论}

本文对飞行器的红外图像进行仿真建模, 由于飞 行器在运动过程中其姿态的变化主要受控制模型的 影响, 因此文中首先建立了飞行器运动与控制模型; 然后研究了飞行器面源热平衡方程, 并应用 CFD 对 飞行器的绝热壁温度场进行了求解; 最后研究了飞行 器绝热壁温度的高度简化算法, 并对飞行器的红外辐 射图像进行了仿真计算。所得到的主要结论有:

1) 飞行器蒙皮的绝热壁温度场可以进行高度简 化处理, 简化后大部分面源的绝热壁温度与 CFD 计 算结果误差在 $3 \mathrm{~K}$ 以内。

2) 飞行器飞行高度在 $20 \mathrm{~km}$ 以下时, 其红外图 像的亮度随着飞行高度的上升而下降, 随着飞行速度 的增加而增加。

3） 8 12 $\mu \mathrm{m}$ 波段的图像亮度明显高于 $3 \sim 5 \mu \mathrm{m}$ 波段。

\section{参考文献：}

[1] 刘娟, 龚光红, 韩亮, 等.飞机红外辐射特性建模与仿真 [J]. 红外与激 光工程, 2011, 40(7): 1219-1213.

LIU Juan, GONG Guanghong, HAN Liang, et al. Modeling and simulation of airplane infrared characteristic[J]. Infrared and Laser Engineering, 2011, 40(7): 1219-1213.

[2] 李建勋, 童中翔, 王超哲, 等. 飞机红外辐射特性分析与易损性评估 [J]. 激光与红外, 2013, 43(2): 180-185.

LI Jianxun, TONG Zhongxiang, WANG Chaozhe, et al. IR radiation characteristics analysis and vulnerability assessment of aircraft[J]. Laser \& Infrared, 2013, 43(2): 180-185.

[3] HUANG Hesong, TONG Zhongxiang, WANG Chaozhe, et al. Numerical and Experimental study of pyrophoric activated metal Mg surface combustion characteristics[J]. Royal Society Open Science, 2018, 5: 172064.

[4] 汪中贤, 史建军, 张正武. 喷气式飞机尾焰红外辐射的理论计算与仿 真 $[\mathrm{J}]$. 火力与指挥控制, 2011, 36(11): 83-86.

WANG Zhaongxian, SHI Jianjun, ZHANG Zhangwu. Theoretical Calculation and Simulation of Infrared Radiation of Jet Aircraft Plume[J]. Fire Control \& Command control, 2011, 36(11): 83-86.

[5] 陈卫, 汪中贤, 马东辉, 等. 非均匀热气体红外辐射特性计算与仿真 [J]. 红外与激光工程, 2010, 39(1): 17-21.
CHEN Wei, WANG Zhongxian, MA Donghui, et al. Calculation and simulation of infrared radiation characteristics of non-uniform hot gas[J]. Infrared and Laser Engineering, 2010, 39(1): 17-21.

[6] 高思莉, 汤心溢. 空中飞行目标尾焰红外辐射信号的建模与仿真 [J]. 光电工程, 2007, 34(8): 25-27.

GAO Sili, TANG Xinyi. Building model of the plume released from the flying machine and simulation [J]. Opto-Electronic Engineering, 2007, 34(8): 25-27.

[7] 范仁钰. 下遮挡二元喷口的气动和红外辐射特性的数值研究 [D]. 南 京: 南京航空航天大学, 2009.

FAN Renyu. Numerical Investigation on the Aerodynamics and Infrared Radiation Characteristics of 2-Dimensional Nozzles with Sheltering Baffles[D]. Nanjing: Nanjing University of Aeronautics and Astronautics, 2009.

[8] 李建勋, 童中翔, 王超哲, 等. 发动机热喷流红外辐射计算与仿真 [J]. 光谱学与光谱分析, 2013, 33(1): 7-13.

LI Jianxun, TONG Zhongxinag, WANG Chaozhe, et al. Calculation and Simulation on Infrared Radiation of Hot Jet from Engine[J]. Spectroscopy and Spectral Analysis, 2013, 33(1): 7-13.

[9] HU Chongwei, Joe Der, Jr, et al. A simple 2D-nozzle plume model for IR analysis[R]. AIAA-80-1808, 1980.

[10] HUANG Hesong, TONG Zhongxiang, CHAI Shijie, et al. Experimental and numerical study of chaff cloud kinetic performance under impact of high-speed airflow[J]. Chinese Journal of Aeronautics, 2018, 31(11): 2080-2092.

[11] 张志波, 童中翔, 王超哲, 等. Lowtran 在红外成像仿真中的应用[J]. 计算机应用与软件, 2013, 30(7): 24-26.

ZHANG Zhibo, TONG Zhongxiang, WANG Chaozhe, et al. The Application of Lowtran in Infrared Imaging Simulation[J].Computer Applications and Software, 2013, 30(7): 24-26.

[12] 吴北贞, 李卫, 陈洪滨, 等. 大气辐射传输实用算法 $[\mathrm{M}]$. 北京: 气象 出版社, 1998.

WU Beiying, LI Wei, CHEN Hongbin, et al. Practical Algorithm of Atmospheric Radiation Transmission[M]. Beijing: Meteorological Publishing House, 1998.

[13] 李刘苗. 海天背景目标红外辐射特性研究[D]. 西安: 西安电子科技大 学, 2011 .

LI Liumiao. Research on Infrared Radiation Characteristic of Object Under Sky and Sea Background[D]. Xi'an: Xidian University, 2011. 